\title{
Metabolic profile of the synthetic drug 4,4'-dimethylaminorex in urine by LC-MS-based techniques: selection of the most suitable markers of its intake
}

\author{
Claudia Chieffi ${ }^{1}$. Cristian Camuto ${ }^{1} \cdot$ Fabio De-Giorgio $^{2,3} \cdot$ Xavier de la Torre $^{1} \cdot$ Francesca Diamanti $^{1}$. \\ Monica Mazzarino ${ }^{1} \cdot$ Claudio Trapella $^{4} \cdot$ Matteo Marti $^{5,6} \cdot$ Francesco Botrè $^{1,7} \mathbb{C}$
}

Received: 11 May 2020 / Accepted: 18 July 2020 / Published online: 19 August 2020

(c) The Author(s) 2020

\begin{abstract}
Purpose In this study, the phase I and II metabolic pathways of 4,4'-dimethylaminorex were characterized to select the marker(s) of intake allowing the unequivocal identification of this novel psychoactive substance in urine samples.

Methods The metabolic profile of 4,4'-dimethylaminorex was characterized using both in vitro and in vivo models. In detail, for the in vitro experiments, either pooled human liver microsomes or recombinant cytochrome P450 isoforms were selected, whereas the in vivo investigation was performed on male mice ICR $\left(\mathrm{CD}-1^{\circledR}\right)$. Sample preparation included enzymatic hydrolysis followed by liquid/liquid extraction. The instrumental analysis was performed by ultra-high-performance liquid chromatography coupled to either high- or low-resolution tandem mass spectrometry.

Results Five metabolic products were isolated only for the cis-isomer: the phase I metabolic reactions included hydrolysis, carboxylation, hydroxylation, and carbonylation. CYP2D6 was the principal isoenzyme involved, and the incubation in the presence of different allelic variants showed significant alteration on the metabolic profile. Once formed, the phase I metabolites underwent extensive conjugation. Not only the most abundant compounds detected, but also those with the most extended window of detection, were the carboxylated and the hydroxylated metabolites. These analytes together with the parent compound were selected as the most suitable markers of intake.

Conclusions Knowledge of the metabolic profiles of the new drugs is essential for their fast identification. Phase I and phase II metabolites of 4,4'-dimethylaminorex were identified and selected as markers of intake, to be considered as the most suitable analytical targets in forensic toxicology.
\end{abstract}

Keywords 4,4'-Dimethylaminorex · Anti-doping analysis $\cdot$ In vitro and in vivo metabolism studies $\cdot$ CYP2D6 $\cdot$ Novel psychoactive substances (NPS) $\cdot$ UHPLC-MS/MS

Claudia Chieffi and Cristian Camuto equally contributed.

Francesco Botrè

francesco.botre@uniroma1.it

1 Laboratorio Antidoping, Federazione Medico Sportiva Italiana, Largo Giulio Onesti, 1, 00197 Rome, Italy

2 Section of Legal Medicine, Department of Health Care Surveillance and Bioetics, Università Cattolica del Sacro Cuore, Rome, Italy

3 Fondazione Policlinico Universitario A. Gemelli IRCCS, Rome, Italy

4 Department of Chemistry and Pharmaceutical Sciences, University of Ferrara, Ferrara, Italy
5 Section of Legal Medicine, Department of Morphology, Surgery and Experimental Medicine, University of Ferrara, Ferrara, Italy

6 Department of Anti-Drug Policies, Collaborative Center for the Italian National Early Warning System, Presidency of the Council of Ministers, Rome, Italy

7 Dipartimento di Medicina Sperimentale, "Sapienza" Università di Roma, Viale Regina Elena 324, 00161 Rome, Italy 


\section{Introduction}

Since the 1960s, new psychoactive substances (NPS), also known as "designer" drugs, became a global problem, which has been continuously expanding [1]. Besides the traditional amphetamine-type designer drugs, which have been widely abused for decades, the number of newly detected psychoactive substances has been increasing dramatically. At present, they include several different classes of drugs of abuse, e.g., cannabimimetics, fentanyls, piperidines, tryptamine derivatives and the groups of phenethylamines [1,2]. These substances are chemically developed from the base structures of known compounds, exploring all possible substitutions to retain the pharmacological effects of the original drugs and at the same time to circumvent the existing laws and their disclosure [3-6].

4,4'-Dimethylaminorex (4-methyl-5-(4-methylphenyl)4,5-dihydro-1,3-oxazol-2-amine, also known as 4,4'DMAR, or simply DMAR) is a synthetic psychostimulant and anorexigenic substance structurally correlated to the controlled drugs aminorex ( $(R S)$-5-phenyl-4,5-dihydro1,3-oxazol-2-amine) and 4-methylaminorex (4-methyl5-phenyl-4,5-dihydro-1,3-oxazol-2-amine, also known as 4-MAR) [7, 8]. Both aminorex and 4-MAR were introduced into the market in 1960s as appetite suppressants, but were subsequently withdrawn due to fatal complications related to pulmonary hypertension $[9,10]$. Due to two chiral centres within the oxazoline ring, 4,4'-DMAR exists in four enantiomers or two different $(( \pm)$-cis and $( \pm)$-trans) racemates.

4,4'-DMAR was identified for the first time in 2012 in seized white powder at a national focal point in the Netherlands [11] and reported to the European Monitoring Centre for Drugs and Drug Addiction (EMCDDA) [7]. In 2013, $4,4^{\prime}$-DMAR was associated with 31 deaths in Europe. For this reason, the Commission on Narcotic Drugs, in 2016, decided to internationally control this new synthetic drug by adding it into Schedule II of the Convention on Psychotropic Substances of 1971 [12, 13]. 4,4'-DMAR was initially sold as a "research chemical" on the illicit Internet market in powder form, under different names (e.g., "4-methylU4Euh", "4-methyl-euphoria", "Serotoni”) [14, 15]. Subsequently, it was found on the "street market" as tablets of various colours and shapes, with logos (e.g., star, cross, cherries, or playboy) similar to those reported on "ecstasy" tablets $[14,15]$.

The pharmacological properties of 4,4'-DMAR are mainly due to its interaction with neurotransmitter transporters, namely the dopamine transporter (DAT), the serotonin transporter (SERT) and the norepinephrine transporter (NET). Studies carried out on rat brain synaptosomes have shown that the cis-isomer acts as a potent, non-selective releaser, leading to an increase in the synaptic cleft concentration of monoamines. Specifically, in comparison to aminorex and 4-MAR, the cis-4,4'-DMAR isomer showed higher activity on SERT. This absence of selectivity is probably linked to the para-substitution of the phenyl ring $[13,16,17]$. Similar studies carried out on the trans-isomer showed that it was a releasing agent at DAT and NET, although less potent than the cis-isomer; nevertheless, at the SERT, it acts as an uptake blocker $[13,17]$.

The desired and adverse effects of 4,4'-DMAR are mainly linked to the increased levels of extracellular neurotransmitters. For indeed, overstimulation of central serotoninergic and dopaminergic systems causes hyperthermia, agitation, nausea and convulsion, whereas the higher norepinephrine release is responsible of cardiovascular toxicity [13, 17]. These unwanted effects were similar to those of the sympathomimetic drugs 3,4-methylenedioxymethamphetamine (MDMA) and mephedrone; consequently, apart from being considered an NPS, 4,4'-DMAR also falls into the section S6 "stimulants" of the list of prohibited substances and methods published by the World Anti-Doping Agency (WADA) [18].

So far, little is known about the pharmacokinetics of 4,4'DMAR and its analogs; Lucchetti et al. [12] identified four metabolites (hydroxylated, carboxylated, deaminated, and 4-methylnorephedrine) of cis-4,4'-DMAR in rat plasma and brain tissues. No information was, instead, available on the urinary excretion profile of the 4,4'-DMAR isomers.

The aim of the present study was to identify and select the most suitable marker(s) of intake of 4,4'-DMAR for forensic purposes. We are here presenting the results of in vitro studies performed with human liver microsomes (HLM) and recombinant cytochrome $\mathrm{P} 450$ isoforms. In vivo metabolism was, instead, explored on male mice ICR $\left(\mathrm{CD}-1^{\circledR}\right)$, to investigate the urinary excretion profile and to establish the windows of detection 4,4'-DMAR and its metabolites.

\section{Materials and methods}

\section{Chemicals and reagents}

cis/trans-4,4'-DMAR were synthesized and provided by the University of Ferrara, Department of Organic Chemistry. $( \pm)$-Amphetamine-D $\mathrm{D}_{11}$ (used as internal standard: ISTD) was provided by Sigma-Aldrich (St. Louis, MO, USA). The reagents (formic acid, acetic acid, ammonium acetate, sodium phosphate, sodium hydrogen phosphate, potassium carbonate, potassium hydrogen carbonate, acetonitrile, methanol, ethyl acetate, chloroform, and tert-butyl methyl ether) were all analytical grade and supplied by Sigma-Aldrich (Milan, Italy). The ultra-purified water used was of Milli-Q-grade (Millipore Italia, Vimodrone, Milan, Italy). The enzymes 
$\beta$-glucuronidase (from E. coli) and arylsulfatase (from Helix pomatia) used for the enzymatic hydrolysis of glucuronoand sulfo-conjugate metabolites were purchased from Roche (Monza, Italy).

The enzymatic proteins (CYP recombinant isoforms CYP1A2, CYP2C19, CYP2C9, CYP3A4, CYP3A5, and CYP2D6) and the HLM, pooled from 20 Caucasian mixed male and female donors of different ages, as well as all the analytically reagents used for the in vitro metabolism studies [sodium phosphate and, magnesium chloride hexahydrate, glucose-6-phosphate, glucose-6-phosphate dehydrogenase, nicotinamide adenine dinucleotide phosphate $\left(\mathrm{NADP}^{+}\right)$and CYP450 oxidoreductase] were supplied by BD Biosciences (Milano, Italy). HLM containing the different CYP2D6 allelic variants (moderate, high, and without activity) were purchased from XenoTech (Kansas City, KS, USA).

\section{Animals}

Male ICR $\left(\mathrm{CD}-1^{\circledR}\right)$ mice weighing $25-30 \mathrm{~g}$, were purchased from Harlan Italy (S. Pietro al Natisone, Italy). Food (Diet 4RF25 GLP; Mucedola, Settimo Milanese, Milan, Italy) and tap water were available ad libitum during the entire time when the animals spent in their home cages. For the in vivo studies, a group of three mice was administered with cis-4,4'-DMAR, dissolved in absolute ethanol (final concentration of $2 \%$ ), and Tween 80 (2\%) and brought to its final volume with saline $(0.9 \% \mathrm{NaCl})$. The solution made with ethanol, Tween 80 , and saline was also used as the vehicle. The drug was administered by intraperitoneal injection at a volume of $4 \mu \mathrm{L} / \mathrm{g}$; the final concentration of $4,4^{\prime}$-DMAR was $10 \mathrm{mg} / \mathrm{kg}$. The control group of three mice was administered only with vehicle solution. The mice were singlehoused (1 mouse per cage, with a per-animal floor area of 80 $\mathrm{cm}^{2}$ and a minimum enclosure height of $12 \mathrm{~cm}$ ) in a colony room under constant temperature $\left(23-24{ }^{\circ} \mathrm{C}\right)$ and humidity $(45-55 \%)$. Urine samples were collected in $2 \mathrm{~mL}$ tubes before and after 2, 4, 6, and $9 \mathrm{~h}$ from the administration of the drug, and stored at $-20^{\circ} \mathrm{C}$ until analysis. Experimental protocols followed in the present study were planned and carried out in compliance to the new European Communities Council Directive of September 2010 (2010/63/EU), a revision of Directive 86/609/EEC, and were approved by the Italian Ministry of Health (license n. 335/2016-PR) and Ethics Committee of the University of Ferrara.

\section{Protocols for the in vitro studies}

All incubation conditions (i.e., substrate concentrations and incubation times) were preliminarily optimized, considering the protocols currently used in our laboratory to perform similar studies [19-25]. Different concentrations of cis and trans-4,4'-DMAR $(5,10,20$, and $30 \mu \mathrm{M})$ and different incubation times $(30,60,120,240,1440 \mathrm{~min})$ were evaluated. The final incubation medium also contained $3.3 \mathrm{mM}$ magnesium chloride, $1.3 \mathrm{mM} \mathrm{NADP}^{+}, 3.3 \mathrm{mM}$ glucose6-phosphate and $0.4 \mathrm{U} / \mathrm{mL}$ glucose-6-phosphate dehydrogenase in a total volume of $250 \mu \mathrm{L}$. Samples were preincubated at physiological temperature $\left(37^{\circ} \mathrm{C}\right)$ with gentle shaking (500 rpm) for $5 \mathrm{~min}$, and the appropriate enzymatic proteins (either HLM or CYP recombinant isoforms) were added to start the phase I reactions. The phase I reactions were stopped by the addition of $250 \mu \mathrm{L}$ of ice-cold acetonitrile, and the proteins were further precipitated, transferring the samples into an ice bath for $5 \mathrm{~min}$. The precipitate was subsequently separated from the supernatant by centrifugation at 21,000 $\mathrm{g}$ at room temperature for $10 \mathrm{~min}$. Negative control samples, containing all reaction mixture components except the enzymatic proteins, were included to monitor the potential non-enzymatic reactions within the incubation period. Each incubation was performed at least in duplicate.

\section{Sample pretreatment}

The optimization of sample pretreatment considered different extraction protocols, i.e., liquid/liquid extraction at different $\mathrm{pH}$ values $(7,9,11)$ and using different organic solvents (tert-butyl methyl ether, ethyl acetate, chloroform). The selected protocol was based on the use of ethyl acetate at alkaline $\mathrm{pH}$. Briefly, on the samples obtained by either in vitro or in vivo experiments, $50 \mu \mathrm{L}$ of the ISTD (final concentration $500 \mathrm{ng} / \mathrm{mL}$ ) and $50 \mu \mathrm{L}$ of the carbonate buffer $(2 \mathrm{M}, \mathrm{pH}>10)$ were added. The $\mathrm{pH}$ value was then adjusted to 11 by adding the most properly volume of $2 \mathrm{~N} \mathrm{NaOH}$ and liquid/liquid extraction was performed with $7 \mathrm{~mL}$ of ethyl acetate for $6 \mathrm{~min}$ on a mechanical shaker. After centrifugation at $3000 \mathrm{rpm}$ for $2 \mathrm{~min}$, the organic layer was transferred into a $10 \mathrm{~mL}$ tube and evaporated to dryness under nitrogen stream at $40{ }^{\circ} \mathrm{C}$. The extraction procedure was repeated twice. The residue was reconstituted in $50 \mu \mathrm{L}$ of mobile phase (initial composition), and an aliquot of $10 \mu \mathrm{L}$ was injected into the liquid chromatography-mass spectrometry (LC-MS) systems for the detection of phase I metabolites.

To the aqueous layer, $200 \mu \mathrm{L}$ of phosphate buffer $(0.8 \mathrm{M}$, $\mathrm{pH} 7.4$ ), $50 \mu \mathrm{L}$ of $\beta$-glucuronidase (from E. coli) and $50 \mu \mathrm{L}$ of the ISTD (final concentration $500 \mathrm{ng} / \mathrm{mL}$ ) were added, and the samples were incubated for $1 \mathrm{~h}$ at $55{ }^{\circ} \mathrm{C}$. After hydrolysis, $50 \mu \mathrm{L}$ of carbonate buffer $(2 \mathrm{M}, \mathrm{pH}>10)$ and $7 \mathrm{~mL}$ of ethyl acetate were added. The liquid/liquid extraction was carried out for $6 \mathrm{~min}$ on a mechanical shaker. After centrifugation at $3000 \mathrm{rpm}$ for $2 \mathrm{~min}$, the organic layer was transferred to a $10 \mathrm{~mL}$ tube and evaporated to dryness under nitrogen stream at $40{ }^{\circ} \mathrm{C}$. The extraction procedure was repeated twice. The residue was reconstituted in $50 \mu \mathrm{L}$ of mobile phase (initial composition), and an aliquot of 10 
$\mu \mathrm{L}$ was injected into the LC-MS systems for the detection of the glucurono-conjugates metabolites.

To the aqueous layer, $800 \mu \mathrm{L}$ of acetate buffer $(2 \mathrm{M}, \mathrm{pH}$ 5), $50 \mu \mathrm{L}$ of arylsulfatase (from Helix pomatia) and $50 \mu \mathrm{L}$ of ISTD (final concentration $500 \mathrm{ng} / \mathrm{mL}$ ) were added, and the samples were incubated for $2 \mathrm{~h}$ at $55^{\circ} \mathrm{C}$. After hydrolysis, $1 \mathrm{~mL}$ of carbonate buffer $(2 \mathrm{M}, \mathrm{pH}>10)$ and $7 \mathrm{~mL}$ of ethyl acetate were added. The liquid/liquid extraction was carried out for 6 min on a mechanical shaker. After centrifugation at $3000 \mathrm{rpm}$ for $2 \mathrm{~min}$, the organic layer was transferred into a $10 \mathrm{~mL}$ tube and evaporated to dryness under nitrogen stream at $40{ }^{\circ} \mathrm{C}$. The extraction procedure was repeated twice. The residue was resuspended in $50 \mu \mathrm{L}$ of mobile phase (initial composition) and an aliquot of $10 \mu \mathrm{L}$ was injected into the LC-MS systems for the detection of the sulfo-conjugate metabolites.

\section{LC-MS conditions}

\section{Untargeted analysis}

An Agilent 1290 Infinity II UHPLC with a binary gradient system and an automatic injector (Agilent Technologies, Cernusco sul Naviglio, Milano, Italy) was used for the chromatographic separation. Reversed-phase liquid chromatography was performed using Agilent Zorbax Eclipse plus C18 column $(100 \times 2.1 \mathrm{~mm}$ i.d., particle size $1.8 \mu \mathrm{m})$. Ultra-purified water (eluent A) and acetonitrile (eluent B), both containing $0.1 \%$ formic acid, were selected as mobile phases. The chosen elution program started at $2 \% \mathrm{~B}$, and was increased to $60 \% \mathrm{~B}$ in $6 \mathrm{~min}$, to $80 \% \mathrm{~B}$ in $2 \mathrm{~min}$, and finally to $100 \% \mathrm{~B}$ in $1 \mathrm{~min}$. The column was flushed for at least $2 \mathrm{~min}$ at $100 \% \mathrm{~B}$ and finally re-equilibrated at $2 \% \mathrm{~B}$ for $2 \mathrm{~min}$. The flow rate was set at $0.4 \mathrm{~mL} / \mathrm{min}$.

High-resolution/high-accuracy measurements were performed on Agilent Technologies 6545 orthogonal acceleration time-of-flight mass spectrometer, equipped with an electrospray ionization (ESI) source operating in positive mode. Nitrogen was used as the drying and nebulizing gas. The drying gas flow was $10 \mathrm{~L} / \mathrm{min}$, and the temperature was set at $320{ }^{\circ} \mathrm{C}$. The nebulizer gas pressure was $45 \mathrm{psi}$. The applied capillary and fragmentor voltages were 3500 and $150 \mathrm{~V}$, respectively. Mass spectra data were collected from $\mathrm{m} / z 100$ to 1000 at 9300 transients per second. All other parameters were automatically optimized by the daily performed instrument autotuning procedure.

The mass calibration was executed daily at the beginning of every analytical session, using a calibration solution provided by the manufacturer. Purine with an $[\mathrm{M}+\mathrm{H}]^{+}$ ion at $m / z 121.0509$ and an Agilent proprietary compound (HP0921) yielding an ion at $m / z 922.0098$ were simultaneously introduced via the second orthogonal sprayer, and these ions were used as internal calibrants along with all the analysis. All aspects of instrumental control, tuning, method setup and parameters, sample injection, and sequence operation were controlled by the Agilent Technologies Mass Hunter software version B.08.00.

\section{Target analysis}

An Agilent 1200 Rapid Resolution Series HPLC pump with a binary gradient system and automatic injector (Agilent Technologies) was used for the chromatographic separation. Reversed-phase liquid chromatography was performed using a C18 column $(150 \times 2.1 \mathrm{~mm}$ i.d., particle size $2.7 \mu \mathrm{m}$ ). Ultra-purified water (mobile phase A) and acetonitrile (mobile phase B), both containing $0.1 \%$ formic acid, were also selected as mobile phases. The gradient program selected started at $2 \% \mathrm{~B}$, increased to $60 \% \mathrm{~B}$ in 6 min followed by hold for $3 \mathrm{~min}$, and finally increased to $100 \%$ in $4 \mathrm{~min}$. The column was flushed for $2 \mathrm{~min}$ at $100 \% \mathrm{~B}$ and finally re-equilibrated at $2 \% \mathrm{~B}$ for $3 \mathrm{~min}$. The flow rate was set to a constant flow rate of $0.25 \mathrm{~mL} / \mathrm{min}$.

Detection was carried out using a triple quadrupole as a mass analyzer (API4000; ABSciex, Monza, Italy) with positive ESI. The capillary and declustering voltages were set at 5000 and $60 \mathrm{~V}$, respectively. The source temperature was set at $500{ }^{\circ} \mathrm{C}$. Curtain gas, ion source gas 1 (auxiliary gas), and ion source gas 2 (nebulizer gas) pressures were set at 25, 35, and 40 psi, respectively. Full scan, product ion scan, and multiple reaction monitoring (MRM) were used as acquisition modes. The collision-induced dissociation (CID) was performed using nitrogen as collision gas at $5.8 \mathrm{mPa}$, obtained from dedicated nitrogen generator system Parker-Balston model 75-A74, giving gas purity 99.5\% (CPS Analitica, Milan, Italy). All aspects of instrument control, method setup parameters, sample injection, and sequence operation were controlled by Analyst software version 1.6.1 (ABSciex).

\section{Results}

\section{LC-MS(/MS) analysis}

\section{Optimization of instrumental conditions}

MS and MS/MS parameters were optimized by infusing the standard solution $(10 \mu \mathrm{g} / \mathrm{mL}$ in methanol) of either cis- or trans-4,4'-DMAR. Full scan experiments were first performed in both positive and negative ionization modes to select the most appropriate molecular ion. The protonated molecular ion was identified using positive ionization at $m / z$ 191; no adducts were detected. The source parameters were optimized to obtain the maximum signal of the protonated molecular ion selected. Product ion scan experiments 
were then performed at different collision energies (15, $20,25,30,40$, and $50 \mathrm{eV}$ ) to investigate the characteristic fragmentation pathways of both cis- and trans isomers. Figure 1a-c reports both the product ion mass spectra, collected at $25 \mathrm{eV}$ (Fig. 1a, b), and the proposed fragmentation pathways (Fig. 1c). The dominant signals at a collision energy of $25 \mathrm{eV}$ were the product ions at $\mathrm{m} / \mathrm{z} 148$ and 131; by increasing the collision energy at $45-50 \mathrm{eV}$, the product ions at $\mathrm{m} / \mathrm{z} 56$ and 91 became the most abundant ones. The product ions at $m / z 148,131$ and 91 were selected as diagnostic ions (structural markers: characteristic portions of the molecular structure that is common to the parent compound and its metabolites) to postulate the molecular structures of the metabolic products generated by both the in vitro and in vivo models.

The chromatographic conditions were optimized based on the elution program used in our laboratory to detect similar compounds. Different gradients were comparatively evaluated to obtain an optimal separation of the metabolites with isomeric molecular ions (e.g., monohydroxylated metabolites) and to obtain acceptable retention times for the parent compounds and their metabolites.

For the parent compounds, satisfactory chromatographic retention was obtained starting at $15 \%$ of the acetonitrile solvent (mobile phase B); the metabolites, on the contrary, under these conditions were not retained or separated. The optimal conditions in terms of chromatographic retention and selectivity were obtained, starting at $2 \%$ of the acetonitrile solvent (mobile phase B) for both the parent compounds and their metabolic products (see Fig. 2a, b for the results).

\section{Optimization of sample pretreatment conditions}

Sample pretreatment was optimized by testing liquid/liquid extraction at different $\mathrm{pH}$ values $(7,9$, and 11$)$ with various organic solvents (i.e., chloroform, tert-butyl methyl ether and ethyl acetate). The parent compounds were extracted with recoveries higher than $70 \%$ at all the $\mathrm{pH}$ and organic solvent evaluated; in contrast, satisfactory recoveries (higher than $70 \%$ ) for the metabolites were obtained using $\mathrm{pH}$ higher than 10 and ethyl acetate as the organic solvent. The liquid/ liquid extraction at $\mathrm{pH} 11$ using ethyl acetate as the organic solvent was the sample pretreatment selected to extract with optimal recoveries for both the parent compounds and their metabolites.

\section{In vitro investigation}

The in vitro phase I metabolism protocol was optimized, starting from the protocols currently used in our laboratory to perform similar studies [19-25]. Different concentrations of the substrates $(5,10,20$, and $30 \mu \mathrm{M})$ and different incubation times $(30,60,120,240,1440 \mathrm{~min})$ were evaluated. The best results were obtained using a substrate concentration of $20 \mu \mathrm{M}$ and an incubation time of $4 \mathrm{~h}$. Figure $2 \mathrm{a}, \mathrm{b}$ reports the extracted ion chromatograms obtained analysing the samples by incubating the two isomers of 4,4'-DMAR separately in the presence and absence of HLM. No metabolites were detected for the trans-isomer, whereas for the cis-isomer, five compounds not detected in the samples incubated without enzymatic proteins (negative control samples) were identified. Small amounts of trans-4,4'-DMAR were also detected, probably due to thermic isomerization. Indeed, the trans-isomer was also detected in the sample incubated in the absence of HLM (Fig. 2a(2)). Table 1 reports the elemental compositions of the five compounds detected in the sample incubated in the presence of HLM. In detail, we have identified for the $c i$ s-isomer (also see Fig. 2a for the extracted ion chromatograms):

- Two chromatographic peaks (M1, M3) presenting precursor ions at $\mathrm{m} / z 207$ and elemental composition $\mathrm{C}_{11} \mathrm{H}_{14} \mathrm{~N}_{2} \mathrm{O}_{2}$. The presence of the product ion at $m / z 164$ (Table 1), which differs $16 \mathrm{Da}$ from the selected diagnostic product ion at $\mathrm{m} / \mathrm{z}, 148$, denoted the presence of a hydroxyl group.

- One chromatographic peak (M2) with precursor ion at $\mathrm{m} / \mathrm{z} 221$ and elemental composition $\mathrm{C}_{11} \mathrm{H}_{12} \mathrm{~N}_{2} \mathrm{O}_{3}$. The presence of the product ion at $\mathrm{m} / \mathrm{z} 178$ which differed $30 \mathrm{Da}$ from the selected diagnostic fragment ion at $\mathrm{m} / \mathrm{z}$ 148 , together with the absence of the fragment ion at $\mathrm{m} / \mathrm{z} 91$ denoted the presence of a carboxyl group on the aromatic ring.

- One chromatographic peak (M4) presenting precursor ion at $m / z, 205$ and elemental composition $\mathrm{C}_{11} \mathrm{H}_{12} \mathrm{~N}_{2} \mathrm{O}_{2}$. The presence of the product ions at $\mathrm{m} / z, 162$ that differed $14 \mathrm{Da}$ from the selected diagnostic fragment ion at $\mathrm{m} / \mathrm{z}$ 148 , denoted the presence of a carbonyl moiety.

- One chromatographic peak (M5), with precursor ion at $\mathrm{m} / \mathrm{z} 166$ and elemental composition $\mathrm{C}_{10} \mathrm{H}_{15} \mathrm{NO}$ denoted the presence of 4-methylnorephedrine, confirming the data reported in the literature [12].

To investigate the main CYP450 isoforms involved in the cis-4,4'-DMAR biotransformation pathways identified, the parent compound was incubated in the presence of CYP1A2, CYP2C19, CYP2C9, CYP3A4, CYP3A5, and CYP2D6 separately at a concentration of $20 \mu \mathrm{M}$ for $4 \mathrm{~h}$. The results obtained showed that the CYP2D6 was the main isoform involved in the phase I metabolism of $c i s-4,4^{\prime}$-DMAR. Since CYP2D6 is an isoenzyme subjected to genetic polymorphism, the potential differences in the metabolic profile of cis-4,4'-DMAR between poor, normal and rapid metabolizers were also evaluated, by incubating the parent compound in the presence of HLM containing different allelic 

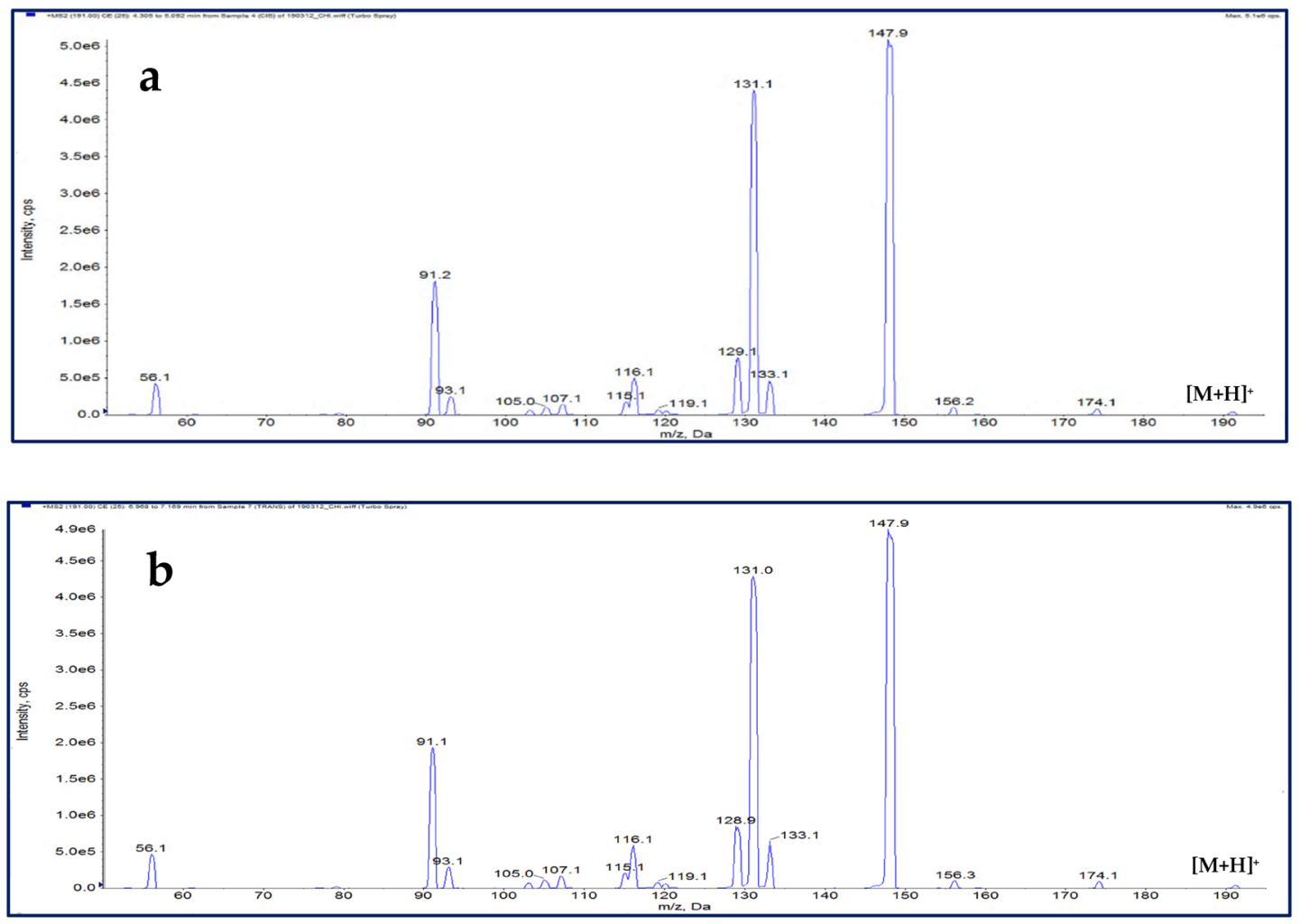

C

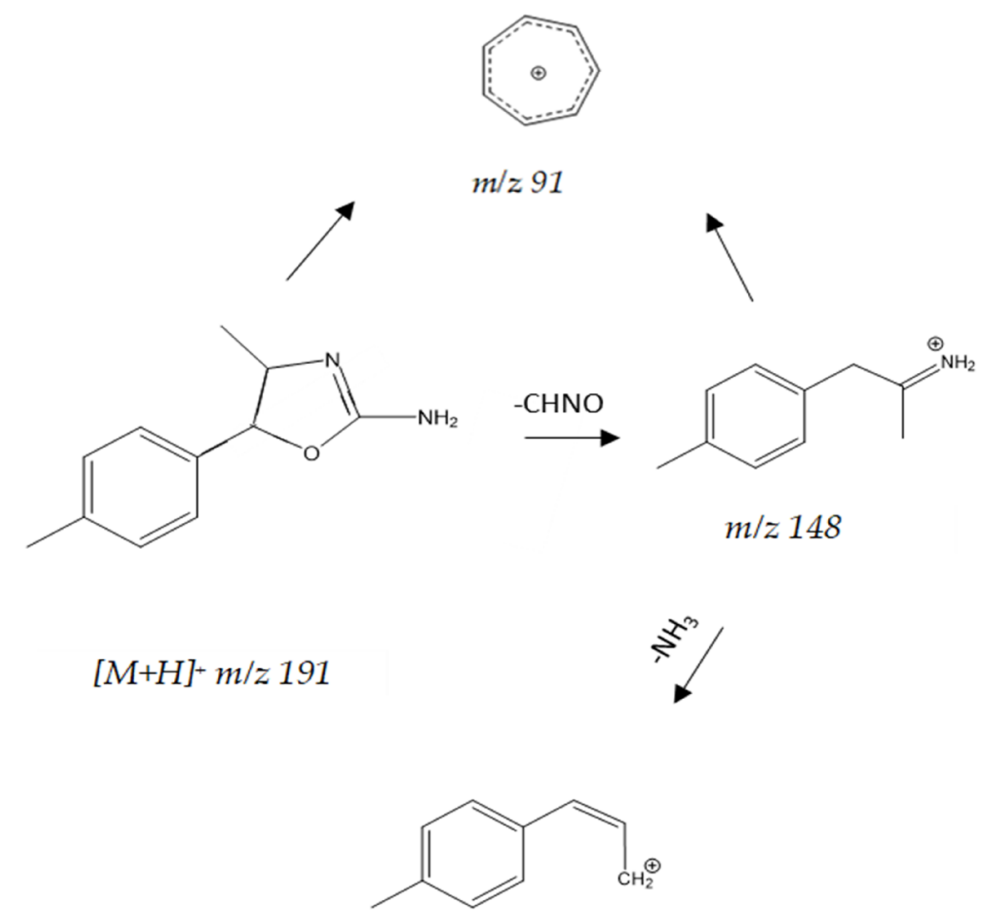

$m / z 131$

Fig. 1 Product ion spectra of $\mathbf{a}$ cis- and $\mathbf{b}$ trans-4,4'-dimethylaminorex (4,4'-DMAR) at a collision energy of $25 \mathrm{eV}$, and proposed fragmentation pathways of both compounds (c) 
a


Fig. 2 a Extracted ion chromatograms obtained analysing the standard solution of cis-4,4'-DMAR at a concentration of $100 \mathrm{ng} / \mathrm{mL}$, $20 \mu \mathrm{M}$ of $c i s-4,4^{\prime}$-DMAR in the presence and absent of human liver microsomes (HLM), using the ultra-high-performance liquid chromatography-quadrupole time-of-flight mass spectrometry (UHPLC- b
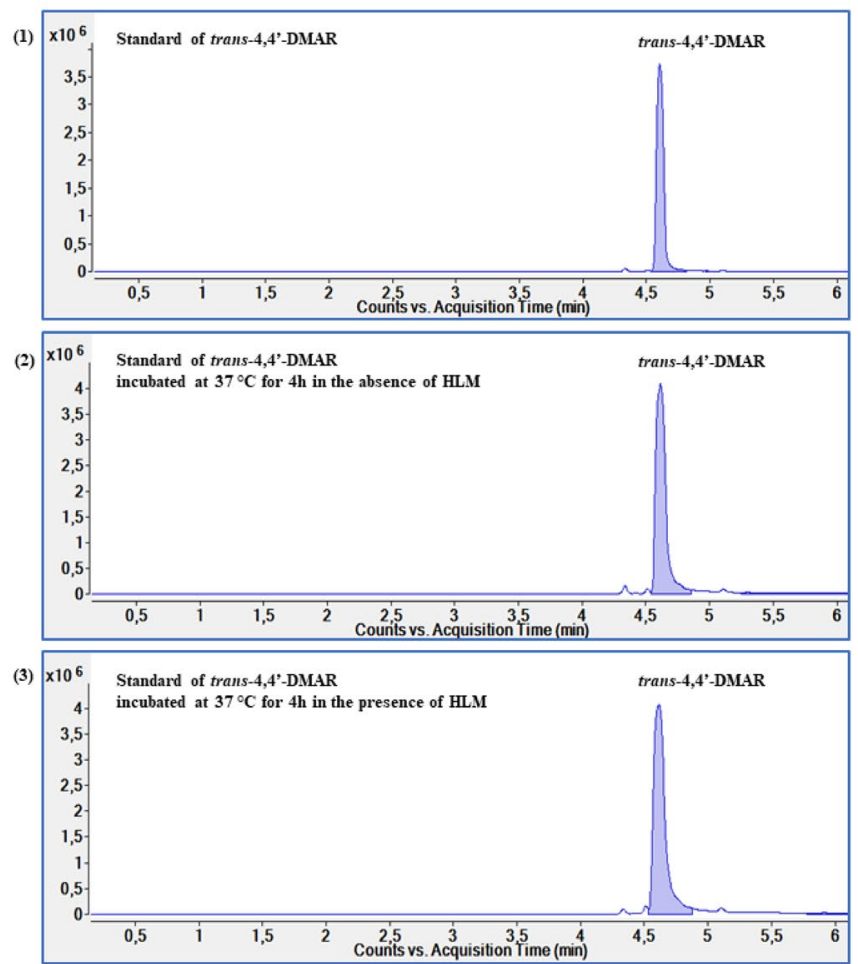

QTOF-MS) system, b Extracted ion chromatograms obtained by analysing the standard solution of cis-4,4'-DMAR at a concentration of $100 \mathrm{ng} / \mathrm{mL}, 20 \mu \mathrm{M}$ of trans-4,4'-DMAR in the presence and absence of HLM, using the UHPLC-QTOF-MS system

Table 1 Elemental compositions, molecular ions, and characteristic product ions of cis-4,4'-dimethylaminorex (4,4'-DMAR) and its metabolites obtained by in vitro experiments using human liver microsomes

\begin{tabular}{|c|c|c|c|c|c|c|c|}
\hline \multirow[t]{2}{*}{ ID } & \multirow[t]{2}{*}{ Metabolic reaction } & \multicolumn{3}{|l|}{ QTOF-MS } & \multicolumn{3}{|l|}{ QqQ-MS } \\
\hline & & Elemental composition & {$\left[\mathrm{M}+\mathrm{H}^{]+}(m / z)\right.$} & Error $(\Delta \mathrm{ppm})$ & $\begin{array}{l}\text { Precursor } \\
\text { ion }(m / z)\end{array}$ & Product ion $(\mathrm{m} / \mathrm{z})$ & Collision energy $(\mathrm{eV})$ \\
\hline M1 & Hydroxylation & $\mathrm{C}_{11} \mathrm{H}_{14} \mathrm{~N}_{2} \mathrm{O}_{2}$ & 207.1179 & 2.89 & 207 & $91,117,147,164$ & $40,40,35,30$ \\
\hline M2 & Carboxylation & $\mathrm{C}_{11} \mathrm{H}_{12} \mathrm{~N}_{2} \mathrm{O}_{3}$ & 221.0921 & 3.22 & 221 & $56,161,178$ & $40,35,30$ \\
\hline M3 & Hydroxylation & $\mathrm{C}_{11} \mathrm{H}_{14} \mathrm{~N}_{2} \mathrm{O}_{2}$ & 207.1179 & 3.86 & 207 & $91,117,147,164$ & $40,40,35,30$ \\
\hline M4 & Carbonylation & $\mathrm{C}_{11} \mathrm{H}_{12} \mathrm{~N}_{2} \mathrm{O}_{2}$ & 205.0972 & 3.16 & 205 & $91,115,145,162$ & $40,40,35,30$ \\
\hline M5 & Hydrolysis & $\mathrm{C}_{10} \mathrm{H}_{15} \mathrm{NO}$ & 166.1226 & 3.39 & 166 & 91,148 & 40,30 \\
\hline $\mathrm{P}$ & cis-4,4'-DMAR & $\mathrm{C}_{11} \mathrm{H}_{14} \mathrm{~N}_{2} \mathrm{O}$ & 191.1179 & 1.23 & 191 & $91,131,148$ & $40,35,30$ \\
\hline
\end{tabular}

QTOF-MS quadrupole time-of-flight-mass spectrometry, $Q q Q-M S$ triple quadrupole-mass spectrometry, $P$ parent

variants of CYP2D6 (high (HA), moderate (MA) and without activity (NA)). Figure 3 shows the percentages of each metabolite referred to the specific allelic variants, except for M5, which was found in trace amounts only. Significant differences in the metabolic profile were registered: M1 was the only metabolite detected after incubation in the presence of CYP2D6 without activity (NA); on the contrary, CYP2D6 with moderate (MA) and high activity (HA) led to the formation of M1, M2, M3, and M4 in different amounts.

\section{In vivo investigation}

Urine samples from three mice, collected before and after 2, 4, 6, and $9 \mathrm{~h}$ from the administration of $10 \mathrm{mg} /$ $\mathrm{kg}$ of $c i s-4,4^{\prime}$-DMAR, were analysed using the analytical 




Fig. 3 cis-4,4'-DMAR, M1, M2, M3, M4 and M5 percentages according to CYP2D6 high activity (HA), moderate activity (MA) and without activity (NA) protocol (sample pretreatment and instrumental analysis) previously developed. Figures 4 and 5 report extracted ion chromatograms obtained by using target analysis for mouse 3 (Fig. 4) and the excretion profile of cis-4,4'DMAR and its main metabolites (M1, M2, and M3) in the free and conjugated fractions for all the three mice studied (Fig. 5). All the metabolites identified by the in vitro analysis were detected, except for M5 (Fig. 4). Briefly, M1 (monohydroxylated metabolite) was present in a high percentage (80-90\%) in the free fraction and in a percentage of $10-20 \%$ in the glucuronide conjugate fraction, whereas no signal was detected in the sulfo-conjugate fraction. M2 (carboxylated metabolite) was, instead, present in lower percentage in the free and glucuronide fraction (lower than $30 \%$ with the exception of mouse 2 , in which the glucuronide was present in higher amount), and more than $70 \%$ as sulfo-conjugate. M3 (monohydroxylated metabolite) was present in a percentage of $40-60 \%$ in both free and

a

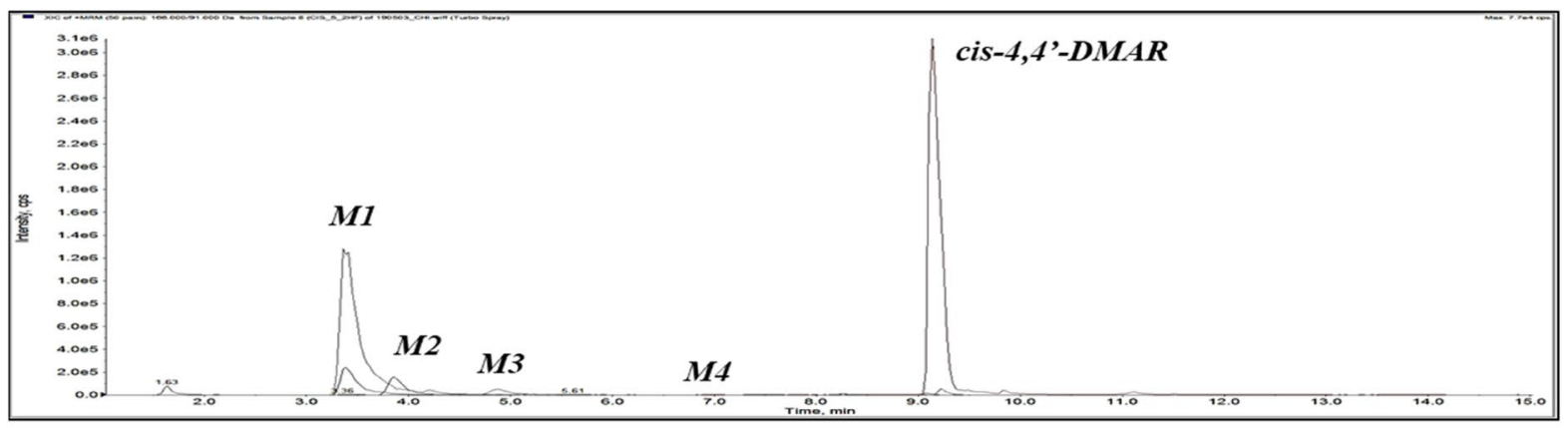

b

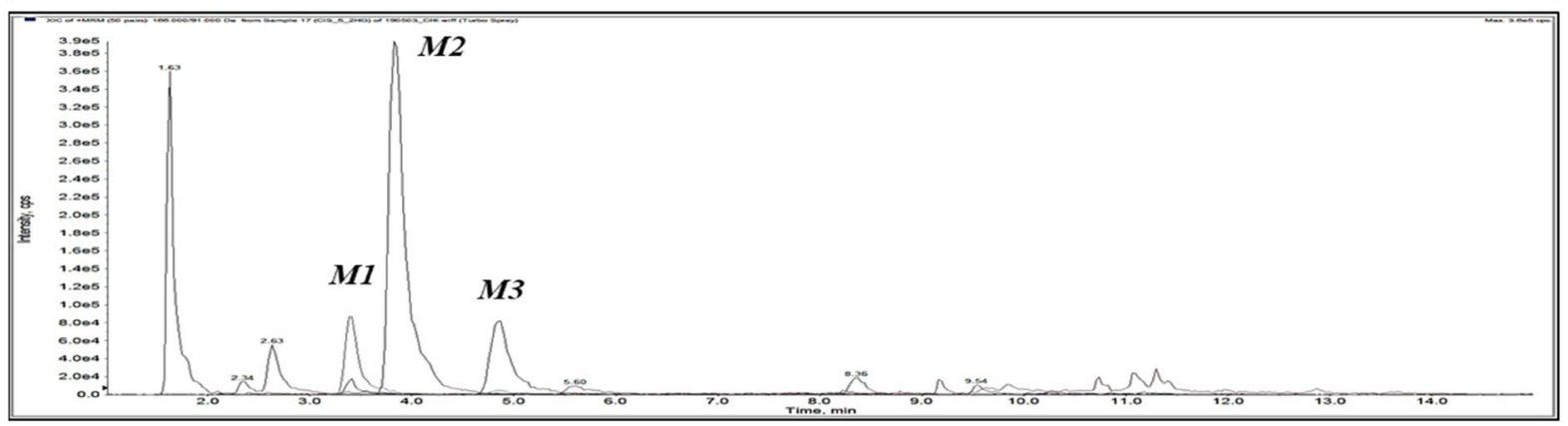

C



Fig. 4 Liquid chromatography-tandem mass spectrometry (LC-MS/ MS) extracted ion chromatograms obtained by analysing the urine collected from a single mouse (number 3 ) after $4 \mathrm{~h}$ from drug admin- istration: a without enzymatic hydrolysis (free fraction), b after hydrolysis with $\beta$-glucuronidase (glucuronide fraction) and $\mathbf{c}$ after hydrolysis with arylsulfatase (sulfate fraction) 

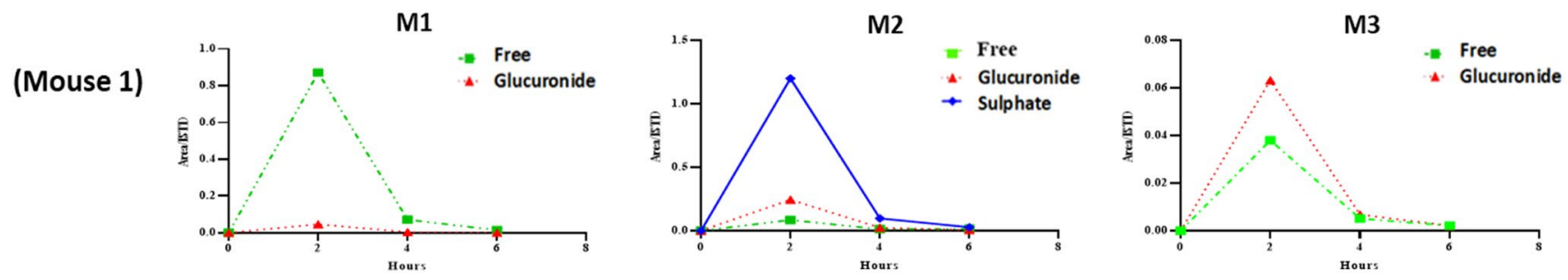

(Mouse 2)
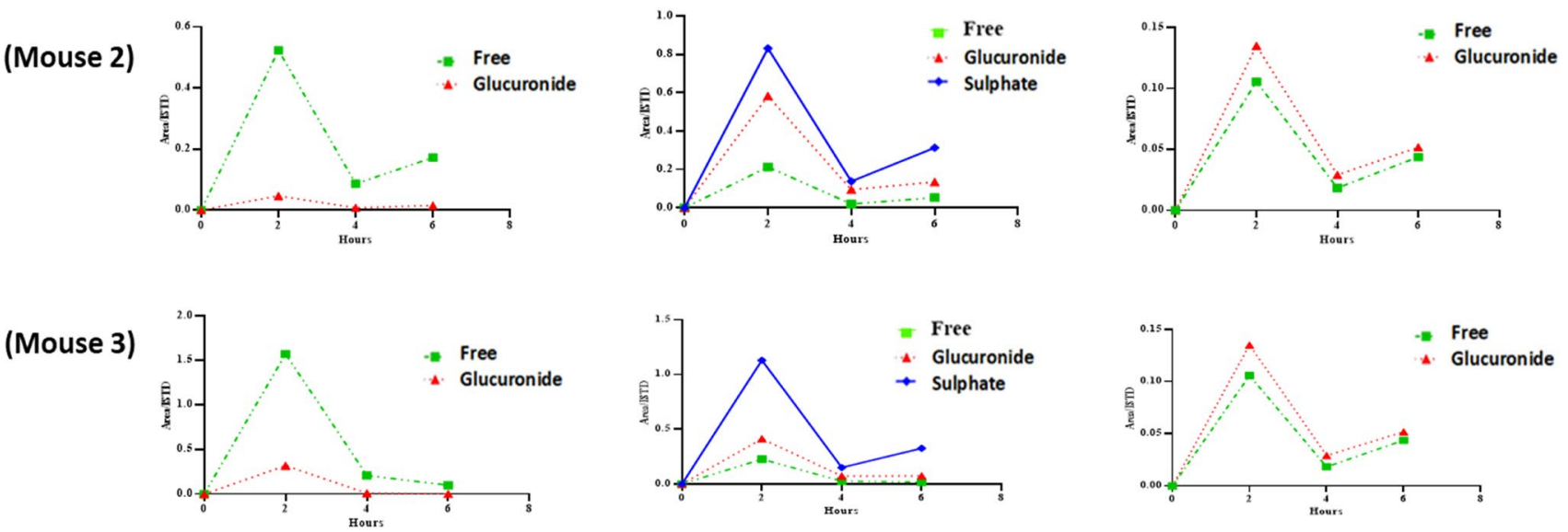

Fig. 5 Excretion profiles of cis-4,4'-DMAR and its main metabolites (M1, M2 and M3) in the free, glucuronide and sulfate forms into mouse urine. ISTD internal standard

(Mouse 1)



(Mouse 2)

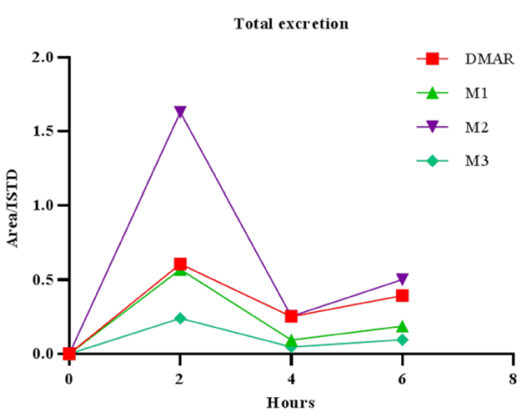

(Mouse 3)



Fig. 6 Total excretion profile (free + glucuronide + sulfate forms) of cis-4,4'-DMAR and its main metabolites (M1, M2 and M3) into mouse urine

glucuronide forms and in less than $1 \%$ as sulfo-conjugate (Fig. 5). M4 (carbonylation product) was found in trace amounts only in the free phase (Fig. 4).

Figure 6 shows the total (free + glucuronide + sulphate fractions) urinary excretion profile in the three mice considered. As can be noticed, all the metabolites reached the maximum excretion after $2 \mathrm{~h}$ from the administration. The metabolites M1 and M2 were the analytes excreted in a higher amount and for a longer time. The parent compound found only in the free fraction was also excreted at high concentration and for all the intervals of time considered.

\section{Discussion}

The main aim of this study was to investigate the phase I and II metabolism of 4,4'-DMAR, with selecting the most appropriate marker(s) of intake allowing the unequivocal detection of this NPS in urine for doping and forensic purposes. Indeed, metabolism studies from previous investigators reported data obtained from blood samples only [12]. Clearly, blood is the primary biological fluid to monitor the levels of an exogenous compound after a 
recent intake, also allowing to know whether the subject is still under its pharmaco-toxicological effects. At the same time, urine is a more appropriate fluid in the case a longer window of detectability is required, targeting either the parent drug or its metabolites.

In this study, we have performed both in vitro studies and in vivo studies on animal models. The use of HLM and recombinant cytochrome $\mathrm{P} 450$ isoforms allowed to reproduce metabolism in humans and characterize the enzymatic isoforms involved in the metabolic pathways detected, whereas the analysis of urine samples collected from mice allowed to define the window of detection of the parent compounds and its main metabolites, and to select the most appropriate target compound(s) to detect drugs after recent intake. Five metabolites (see Fig. 7 for the proposed molecular structures) were detected after incubation of the cis-isomer with HLM, and four metabolites in the mice urine samples. The metabolic reactions detected are typical of amphetamine-like compounds: hydroxylation, carboxylation and carbonylation, confirming in part the data previously reported in blood by Lucchetti et al. [12]. The metabolite originating from hydrolysis, the paramethyl derivative of norephedrine, was instead detected only after incubation of 4,4'-DMAR in the presence of HLM.

The main isoenzyme involved in the phase I metabolism of 4,4'-DMAR was CYP2D6, and significant metabolic differences were observed after incubation in the presence of different allelic variants of this isoenzyme, indicating significant differences in the excretion profile of 4,4'-DMAR between poor, normal and rapid metabolizers.
The phase I metabolites once formed were extensively conjugated in vivo, and the use of both $\beta$-glucuronidase and arylsulfatase to perform the enzymatic hydrolysis of phase II metabolites during the sample pretreatment step is recommended, being the carboxylated metabolites excreted in urine mainly as sulfo-conjugate and the hydroxylated metabolites, or, only as glucurono-conjugate.

Carboxylation (M2) and the aromatic hydroxylation (M1) were likely to be the major metabolic pathways identified. These metabolites were selected as the most suitable markers of intake. In addition, because the significant alteration in the formation of the metabolites by the different allelic variants of the isoenzyme CYP2D6 is involved in the phase I metabolism of $c i s-4,4^{\prime}$-DMAR, the selection of the parent compound together with the metabolites M1 and M2 might be useful for more sensitive assessment of cis-4,4'-DMAR intake in humans.

Concerning the trans-isomer, no metabolites were detected neither in vitro nor in vivo studies indicating a stereoselectivity in the metabolism of 4,4'-DMAR. Indeed, as reported in previous studies, metabolizing enzymes might display remarkable differences in drug metabolism. As is well known for pharmaceutical drugs, the pharmacokinetic profile can be significantly different among the stereoisomers of the same drug [26]. This was observed for phase I metabolism catalyzed by CYP isoforms that show stereoselective preference for a specific isomer [26-29] and also for phase II reactions (e.g., sulfation and glucuronidation) [26, $30,31]$. The same stereospecific interactions were described for other drugs of abuse (e.g., methadone, amphetamine) $[32,33]$. These previous studies might explain the metabolic

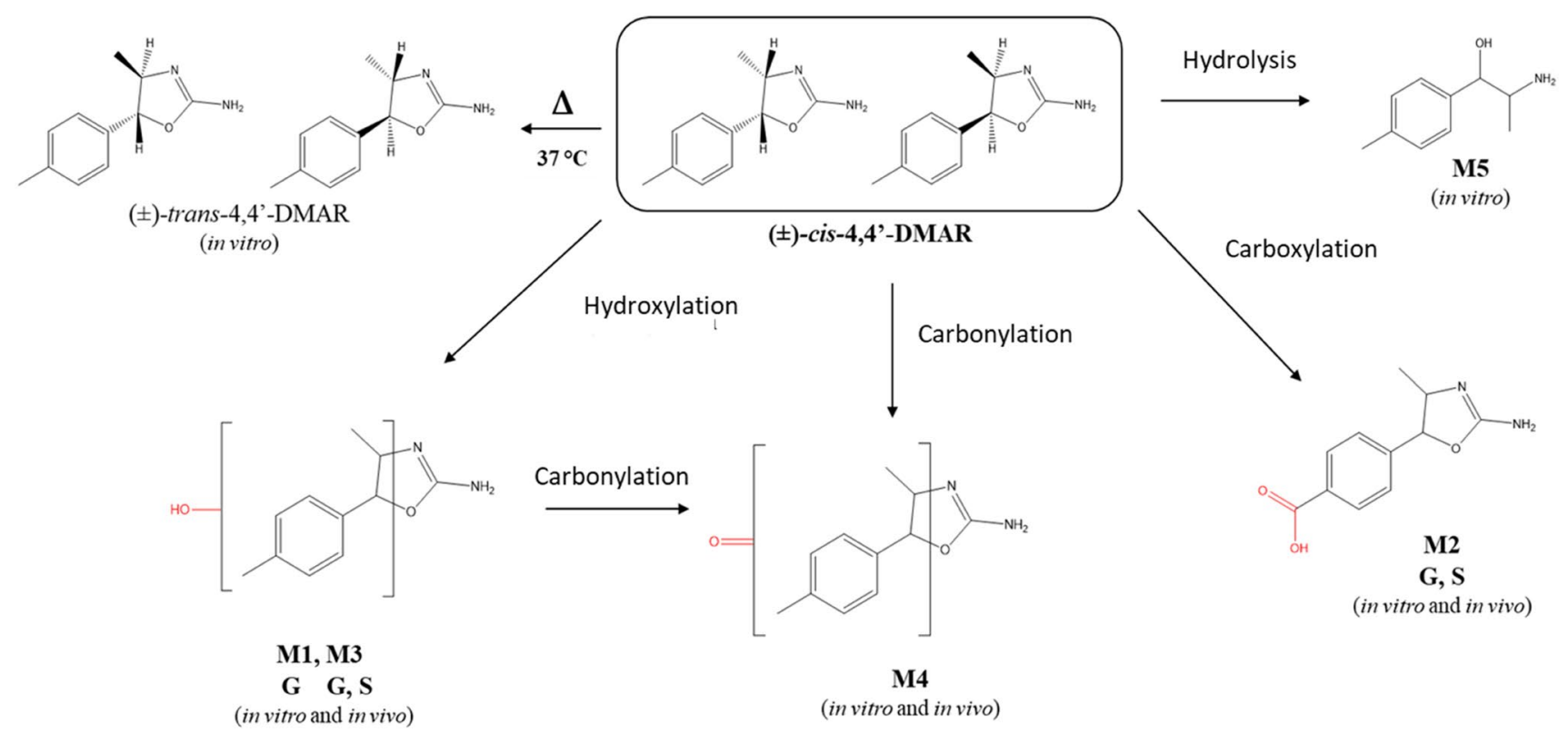

Fig. 7 Scheme of the proposed phase I and II metabolic pathways of cis-4,4-DMAR. $G$ glucorono-conjugate, $S$ sulfo-conjugate 
difference observed between cis- and trans-4,4'-DMAR. Further studies are required to clarify any interactions between the two stereoisomers when administered simultaneously.

\section{Conclusions}

To our knowledge, this work represents the first extensive report of the phase I and II metabolism of 4,4'-DMAR, a synthetic psychostimulant and anorexigenic substance structurally correlated to the controlled drugs aminorex and 4-methylaminorex. The metabolic profile of 4,4'-DMAR in HLM and in urine samples collected from male mice was investigated and the major metabolic pathways identified by LC-MS-based techniques. The trans-isomer resulted to be metabolically stable in our experimental conditions. On the contrary, the cis-isomer was quickly metabolized: 5 metabolites were detected after incubation with HLM and 4 in the urine samples. The compounds selected as most suitable markers of intake were the carboxylated and the hydroxylated metabolites.

In the future, we aim to synthesize the isolated metabolites, to confirm the postulated molecular structures and to shed further light on their pharmacological activities.

Acknowledgements This research was funded by the Drug Policies Department, Presidency of the Council of Ministers, Italy (project: "Effects of NPS: development of a multicentre research for the information enhancement of the Early Warning System" to M. Marti), local funding from the University of Ferrara (FAR 2016 FAR 2017 and FAR 2019 to M. Marti) and the Università Cattolica del Sacro Cuore of Rome (Fondi Linea D1), as well as FIRB 2012 from the Italian Ministry of Education, University and Research (Grant no. RBFR12LDOW to F. De-Giorgio). The project was conducted in agreement with the Presidency of the Council of Ministers-DPA Anti-drug Policies (Italy).

\section{Compliance with ethical standards}

Conflict of interest The authors declare that they have no conflict of interest.

Ethical approval This research involves animals; all applicable international, national, and institutional guidelines for the care and use of animals were followed. All procedures performed in the studies involving animals were in accordance with the ethical standards of the institution or practice at which the studies were conducted. According to the ARRIVE guidelines, all possible efforts were made to minimize the number of animals used, and to minimize the animals' pain and discomfort.

Open Access This article is licensed under a Creative Commons Attribution 4.0 International License, which permits use, sharing, adaptation, distribution and reproduction in any medium or format, as long as you give appropriate credit to the original author(s) and the source, provide a link to the Creative Commons licence, and indicate if changes were made. The images or other third party material in this article are included in the article's Creative Commons licence, unless indicated otherwise in a credit line to the material. If material is not included in the article's Creative Commons licence and your intended use is not permitted by statutory regulation or exceeds the permitted use, you will need to obtain permission directly from the copyright holder. To view a copy of this licence, visit http://creativecommons.org/licenses/by/4.0/.

\section{References}

1. Liechti ME (2015) Novel psychoactive substances (designer drugs): overview and pharmacology of modulators of monoamine signaling. Swiss Med Wkly 145:w14043. https://doi.org/10.4414/ smw.2015.14043 (open access article)

2. Chavant F, Boucher A, Le Boisselier R, Deheul S, Debruyne D (2015) New synthetic drugs in addictovigilance. Therapie 70:167-189. https://doi.org/10.2515/therapie/2015001 (open access article)

3. Dargan PI, Wood DM (eds) (2013) Novel psychoactive substances: classification, pharmacology and toxicology, 1 st edn. Elsevier Inc., Amsterdam

4. Musselman ME, Hampton JP (2014) "Not for human consumption": a review of emerging designer drugs. Pharmacotherapy 34:745-757. https://doi.org/10.1002/phar.1424 (open access article)

5. Fu S, Stojanovska N (2013) Designer drugs. In: Siegal JA, Saukko PJ, Houck MM (eds) Encyclopedia of forensic sciences, 2nd edn. Elsevier, Amsterdam, pp 36-44

6. Meyer MR (2016) New psychoactive substances: an overview of recent publications on their toxicodynamics and toxicokinetics. Arch Toxicol 90:2421-2444. https://doi.org/10.1007/s0020 4-016-1812-x

7. EMCDDA-Europol (2014) EMCDDA-Europol Joint Report on a new psychoactive substance: 4,4'-DMAR (4-methyl5-(4-methylphenyl)-4,5-dihydrooxazol-2-amine). https://doi. org/10.2810/51897

8. Coppola M, Mondola R (2015) 4,4'-DMAR: chemistry, pharmacology and toxicology of a new synthetic stimulant of abuse. Basic Clin Pharmacol Toxicol 117:26-30. https://doi. org/10.1111/bcpt.12399

9. Brandt SD, Baumann MH, Partilla JS, Kavanagh PV, Power JD, Talbot B, Twamley B, Mahony O, O'Brien J, Elliott SP, Archer RP, Patrick J, Singh K, Dempster NM, Cosbey SH (2014) Characterization of a novel and potentially lethal designer drug ( \pm )-cis-para-methyl-4-methylaminorex (4,4'-DMAR, or 'Serotoni'). Drug Test Anal 6:684-695. https://doi.org/10.1002/ dta. 1668

10. McGee M, Whitehead N, Martin J, Collins N (2018) Drugassociated pulmonary arterial hypertension. Clin Toxicol 56:801-809. https://doi.org/10.1080/15563650.2018.1447119

11. Lucchetti J, Marzo CM, Di Clemente A, Cervo L, Gobbi M (2017) A validated, sensitive HPLC-MS/MS method for quantification of cis-para-methyl-4-methylaminorex (cis-4,4'-DMAR) in rat and human plasma: application to pharmacokinetic studies in rats. Drug Test Anal 9:870-879. https://doi.org/10.1002/ dta. 2052

12. Lucchetti J, Marzo CM, Passoni A, Di Clemente A, Moro F, Bagnati R, Gobbi M, Cervo L (2017) Brain disposition of cis-paramethyl-4-methylaminorex (cis-4,4'-DMAR) and its potential metabolites after acute and chronic treatment in rats: correlation with central behavioral effects. J Pharmacol Exp Ther 361:492500. https://doi.org/10.1124/jpet.117.240788 (open access article)

13. Maier J, Mayer FP, Luethi D, Holy M, Jäntsch K, Reither H, Hirtler L, Hoener MC, Liechti ME, Pifl C, Brandt SD, Sitte HH (2018) The psychostimulant ( \pm )-cis-4,4'-dimethylaminorex 
(4,4'-DMAR) interacts with human plasmalemmal and vesicular monoamine transporters. Neuropharmacology 138:282-291. https ://doi.org/10.1016/j.neuropharm.2018.06.018

14. Glanville J, Dargan PI, Wood DM (2015) 4-Methyl-5-(4methylphenyl)-4,5-dihydrooxazol-2-amine (4,4-DMAR, 4,4-dimethylaminorex): availability, prevalence of use, desired effects and acute toxicity. Hum Psychopharmacol 30:193-198. https://doi.org/10.1002/hup.2472

15. Loi B, Zloh M, De Luca MA, Pintori N, Corkery J, Schifano F (2017) 4,4'-Dimethylaminorex (“4,4'-DMAR”; "Serotoni”) misuse: a web-based study. Hum Psychopharmacol 32:e2575. https ://doi.org/10.1002/hup.2575 (open access article)

16. Rickli A, Kolaczynska K, Hoener MC, Liechti ME (2019) Pharmacological characterization of the aminorex analogs 4-MAR, 4,4'-DMAR, and 3,4-DMAR. Neurotoxicology 72:95-100. https ://doi.org/10.1016/j.neuro.2019.02.011

17. WHO (2015) para-Methyl-4-methylaminorex (4,4'-DMAR): critical review report. https://www.who.int/medicines/access/contr olled-substances/5.5_44_DMAR_CRev.pdf?ua=1. Accessed 20 Mar 2020

18. World Antidoping Agency (2020) The world anti-doping code. The 2020 prohibited list international standard. Montreal, Canada. https://www.wada-ama.org. Accessed 27 Mar 2020

19. Mazzarino M, De la Torre X, Di Santo R, Fiacco I, Rosi F, Botrè F (2010) Mass spectrometric characterization of tamoxifene metabolites in human urine using different scan parameters on liquid chromatography/tandem mass spectrometry. Rapid Commun Mass Spectrom 24:749-760. https://doi.org/10.1002/rcm.4432

20. Mazzarino M, Buccilli V, de la Torre X, Fiacco I, Palermo A, Ughi D, Botrè F (2017) Characterization of the phase I and phase II metabolic profile of tolvaptan by in vitro studies and liquid chromatography-mass spectrometry profiling: relevance to doping control analysis. J Pharm Biomed Anal 145:555-568. https://doi. org/10.1016/j.jpba.2017.06.054

21. Mazzarino M, de la Torre X, Botrè F (2014) A liquid chromatography-mass spectrometry method based on class characteristic fragmentation pathways to detect the class of indole-derivative synthetic cannabinoids in biological samples. Anal Chim Acta 837:70-82. https://doi.org/10.1016/j.aca.2014.06.003

22. Mazzarino M, de la Torre X, Fiacco I, Palermo A, Botrè F (2014) Drug-drug interaction and doping, part 1: an in vitro study on the effect of non-prohibited drugs on the phase I metabolic profile of toremifene. Drug Test Anal 6:482-491. https://doi.org/10.1002/ dta. 1592

23. Mazzarino M, de la Torre X, Fiacco I, Botrè F (2014) Drug-drug interaction and doping, part 2: an in vitro study on the effect of non-prohibited drugs on the phase I metabolic profile of stanozolol. Drug Test Anal 6:969-977. https://doi.org/10.1002/dta.1608

24. Mazzarino M, Biava M, de la Torre X, Fiacco I, Botrè F (2013) Characterization of the biotransformation pathways of clomiphene, tamoxifen and toremifene as assessed by LC-MS/ (MS) following in vitro and excretion studies. Anal Bioanal Chem 405:5467-5487. https://doi.org/10.1007/s00216-013-6961-7

25. Mazzarino M, Rizzato $\mathrm{N}$, Stacchini $\mathrm{C}$, de la Torre $\mathrm{X}$, Botrè $\mathrm{F}$ (2018) A further insight into the metabolic profile of the nuclear receptor Rev-erb agonist, SR9009. Drug Test Anal 10:1670-1681. https://doi.org/10.1002/dta.2538

26. Lu H (2007) Stereoselectivity in drug metabolism. Expert Opin Drug Metab Toxicol 3:149-158. https://doi.org/10.1517/17425 255.3.2.149

27. Kaminsky LS, Zhang ZY (1997) Human P450 metabolism of warfarin. Pharmacol Ther 73(1):67-74. https://doi.org/10.1016/s0163 -7258(96)00140-4

28. Rettie AE, Korzekwa KR, Kunze KL, Lawrence RF, Eddy AC, Aoyama T, Gelboin HV, Gonzalez FJ, Trager WF (1992) Hydroxylation of warfarin by human cDNA-expressed cytochrome P-450: a role for $\mathrm{P}-4502 \mathrm{C} 9$ in the etiology of (S)-warfarin-drug interactions. Chem Res Toxicol 5:54-59. https://doi.org/10.1021/tx000 $25 \mathrm{a} 009$

29. Thijssen HH, Flinois JP, Beaune PH (2000) Cytochrome P4502C9 is the principal catalyst of racemic acenocoumarol hydroxylation reactions in human liver microsomes. Drug Metab Dispos 28:1284-1290 (PMID: 11038154)

30. Sten T, Qvisen S, Uutela P, Luukkanen L, Kostiainen R, Finel M (2006) Prominent but reverse stereoselectivity in propranolol glucuronidation by human UDP-glucuronosyltransferases 1A9 and 1A10. Drug Metab Dispos 34:1488-1494. https://doi.org/10.1124/ dmd.106.010371

31. Hartman AP, Wilson AA, Wilson HM, Aberg G, Falany CN, Walle T (1998) Enantioselective sulfation of beta 2-receptor agonists by the human intestine and the recombinant $\mathrm{M}$-form phenolsulfotransferase. Chirality 10:800-803. https://doi.org/10.1002/ (SICI)1520-636X(1998)10:9<800:AID-CHIR4>3.0.CO;2-V

32. Wang J-S, DeVane CL (2003) Involvement of CYP3A4, CYP2C8, and CYP2D6 in the metabolism of $(R)$ - and $(S)$-methadone in vitro. Drug Metab Dispos 31:742-747. https://doi.org/10.1124/ dmd.31.6.742

33. Gunaratna C, Kissinger PT (1998) Investigation of stereoselective metabolism of amphetamine in rat liver microsomes by microdialysis and liquid chromatography with precolumn chiral derivatization. J Chromatogr A 828:95-103. https://doi.org/10.1016/ s0021-9673(98)00822-X

Publisher's Note Springer Nature remains neutral with regard to jurisdictional claims in published maps and institutional affiliations. 